\title{
Designing and Piloting a Specimen Transport System in Burkina Faso
}

Emilie Dama, Abdoulaye Nikiema, Kameko Nichols, Brice Wilfried Bicaba, Souleymane Porgho, Rebecca Greco Koné, Zekiba Tarnagda, Assana Cissé, Irene Ngendakumana, Aimé Adjami, Isaïe Medah, Flavien Ake, and Sara A. Mirza

Efficient specimen transport systems are critical for early disease detection and reporting by laboratory networks. In Burkina Faso, centralized reference laboratories receive specimens from multiple surveillance sites for testing, but transport methods vary, resulting in potential delays and risk to specimen quality. The ministry of health and partners, under the Global Health Security Agenda implementation, piloted a specimen transport system for severe acute respiratory illness (SARI) surveillance in 4 Burkina Faso districts. A baseline assessment was conducted of the current specimen transport network structure and key stakeholders. Assessment results and guidelines for processing SARI specimens informed the pilot specimen transport system design and implementation. Monitoring and evaluation performance indicators included: proportion of packages delivered, timeliness, and quality of courier services (missed or damaged packages). Our baseline assessment found that laboratorians routinely carried specimens from the health center to reference laboratories, resulting in time away from laboratory duties and potential specimen delays or loss of quality. The pilot specimen transport system design engaged Sonapost, the national postal service, to transport specimens from SARI sites to the influenza national reference laboratory. From May 2017 to December 2018, the specimen transport system transported 557 packages containing 1,158 SARI specimens; 95\% (529/557) were delivered within 24 hours of pick-up and 77\% (892/1,158) within 48 hours of collection. No packages were lost. This article highlights lessons learned that may be useful for other countries considering establishment of a specimen transport system to strengthen laboratory system infrastructure in global health security implementation.

Emilie Dama, PhD, is Senior Laboratory Advisor; Rebecca Greco Koné, MPH, is Country Director, Burkina Faso; and Irene Ngendakumana, MD, is Technical Advisor, Eagle Global Services, a CDC contractor; all with the Division of Global Health Protection, Country Office Burkina Faso, Center for Global Health, US Centers for Disease Control and Prevention, Ouagadougou, Burkina Faso. Abdoulaye Nikièma, PharmD, MSc, is Senior Laboratory Specialist and Global Health Security Program Manager for Burkina, African Society for Laboratory Medicine, Ouagadougou, Burkina Faso. Kameko Nichols is a Transport and Logistics Consultant, The Nichols Group LLC, Washington, DC. Brice Wilfried Bicaba, MD, MSc, is an Epidemiologist and Director; Souleymane Porgho, MSc, is an Epidemiologist and Data Manager; and Isaïe Medah, MD, is Director; all at Direction de la Protection de la Santé de la Population, Ministère de la Santé, Ouagadougou, Burkina Faso. Zékiba Tarnagda, DVM, PhD, is Head of Influenza, and Assana Cissé, MSc, is a Bacteriologist and Virologist, Influenza; both at the National Reference Laboratory, Institut de Recherche en Sciences de la Santé, Bobo-Dioulasso, Burkina Faso. Aimé Adjami, PhD, is a Biologist and Executive Director, and Flavien Ake, PhD, is Director; both at DAVYCAS International, Ouagadougou, Burkina Faso. Sara A. Mirza, PhD, is an Epidemiologist, US Centers for Disease Control and Prevention, Division of Bacterial Diseases, National Center for Immunizations and Respiratory Diseases, Atlanta, GA. The findings and conclusions in this report are those of the authors and do not necessarily represent the official position of the Centers for Disease Control and Prevention. 
Keywords: Laboratory capacity, Specimen transport system, Laboratory systems, Global health security, Low-resource settings

$\mathrm{T}$ he International Health Regulations (IHR 2005), in alignment with the Global Health Security Agenda (GHSA), require countries to establish a national laboratory network with real-time surveillance to detect and respond to disease threats. ${ }^{1-3}$ In Burkina Faso, reference laboratories capable of detecting infectious disease threats are centralized in the 2 major cities, Ouagadougou and Bobo-Dioulasso. An effective laboratory network requires a robust and rapid specimen transport system. In resourcelimited settings like Burkina Faso, laboratory system strengthening is challenging and requires innovative solutions to address obstacles. ${ }^{4,5}$ In addition, establishing a specimen transport system requires the commitment and leadership of government and strong coordination and collaboration among donors and partners. The 2015 World Health Organization (WHO) guidance for the transport of infectious substances recommends a comprehensive system to transport specimens safely and reliably. ${ }^{6}$ This system should include transport mechanisms and equipment to move specimens between locations safely and also consider logistics, results reporting, trained personnel, monitoring and evaluation, a policy framework, and standard operating procedures (SOPs). ${ }^{7}$

Burkina Faso has annual outbreaks of endemic diseases, such as dengue and meningitis; the main strategy to monitor and address these diseases is laboratory-based disease surveillance at health facilities. ${ }^{8-12}$ Burkina Faso introduced the WHO Regional Office for Africa (WHO AFRO) Integrated Disease Surveillance and Response (IDSR) system in 2002 and revised it in 2012 to increase the number of priority diseases for public health surveillance. As of 2019, the ministry of health monitors 52 diseases via national surveillance systems, including Ebola, meningitis, cholera, arboviruses, dengue, and severe acute respiratory illnesses such as influenza.

In 2016, the ministry of health and its partners, the African Society for Laboratory Medicine (ASLM) and Davycas International (a local nongovernmental organization), with support from the US Centers for Disease Control and Prevention (CDC) under the GHSA, established sentinel surveillance for severe acute respiratory infections (SARI) in 4 district hospitals. The goal of this SARI surveillance was to obtain epidemiologic and laboratory data on circulating respiratory pathogens in Burkina Faso and build capacity of human resources and laboratory facilities.

Epidemiologic data and SARI specimens were collected from consenting patients. The influenza national reference laboratory (NRL), located in Bobo-Dioulasso in the southwest of the country, was the designated reference laboratory for specimen testing. Sites were selected by the ministry of health based on geographical representativeness and prior successful participation in disease surveillance programs (Figure 1). To meet global influenza surveillance standards aimed at preserving specimen quality, specimens needed to arrive in the influenza NRL for processing within 24 to 48 hours of collection and at $4^{\circ} \mathrm{C}$, and they were required to be triple packaged to meet other specifications in the WHO guidelines for shipment of infectious substances. $^{6,7,13}$

We describe the process of designing and implementing a specimen transport system for SARI surveillance in Burkina Faso with the potential for future expansion to other disease surveillance systems. Lessons learned in Burkina Faso may have relevance for other countries considering the introduction of a specimen transport system.

\section{Methods}

\section{Baseline Assessment Phase}

As a GHSA implementing partner, the ASLM worked to assess and strengthen the specimen referral networks, including specimen transport systems, in CDC's 17 global health security partner countries. ${ }^{3}$ In June 2016, they conducted an assessment of current specimen referral networks in Burkina Faso with the goal of providing recommendations for the design and implementation of the SARI surveillance specimen transport system.

An ASLM consultant and a specimen referral expert conducted semistructured interviews with key stakeholders. The questionnaire was designed to collect information on existing specimen transport systems and related capabilities, focusing on the following areas:

1. Landscape (partners and donors, policy, roles and responsibilities, coordination and communication, and biosafety)

2. Referral network structure (structure, coverage, sample delivery method, optimization of routes, general transport infrastructure)

3. Cost considerations (maintenance, private courier costs, public transport costs)

4. Multisector engagement (courier services, pharmaceuticals, outsourcing, guidelines)

The specimen referral expert interviewed key personnel in the ministry of health, including staff within the Directorate for the Protection of the Population (DPSP), the lead for all disease surveillance activities; the Directorate of Biomedical Laboratories (DLBM), the lead for all national laboratories; and the national vaccination program (DPV). Laboratory and clinical staff from health facilities and laboratory personnel and leaders at the influenza NRL were 


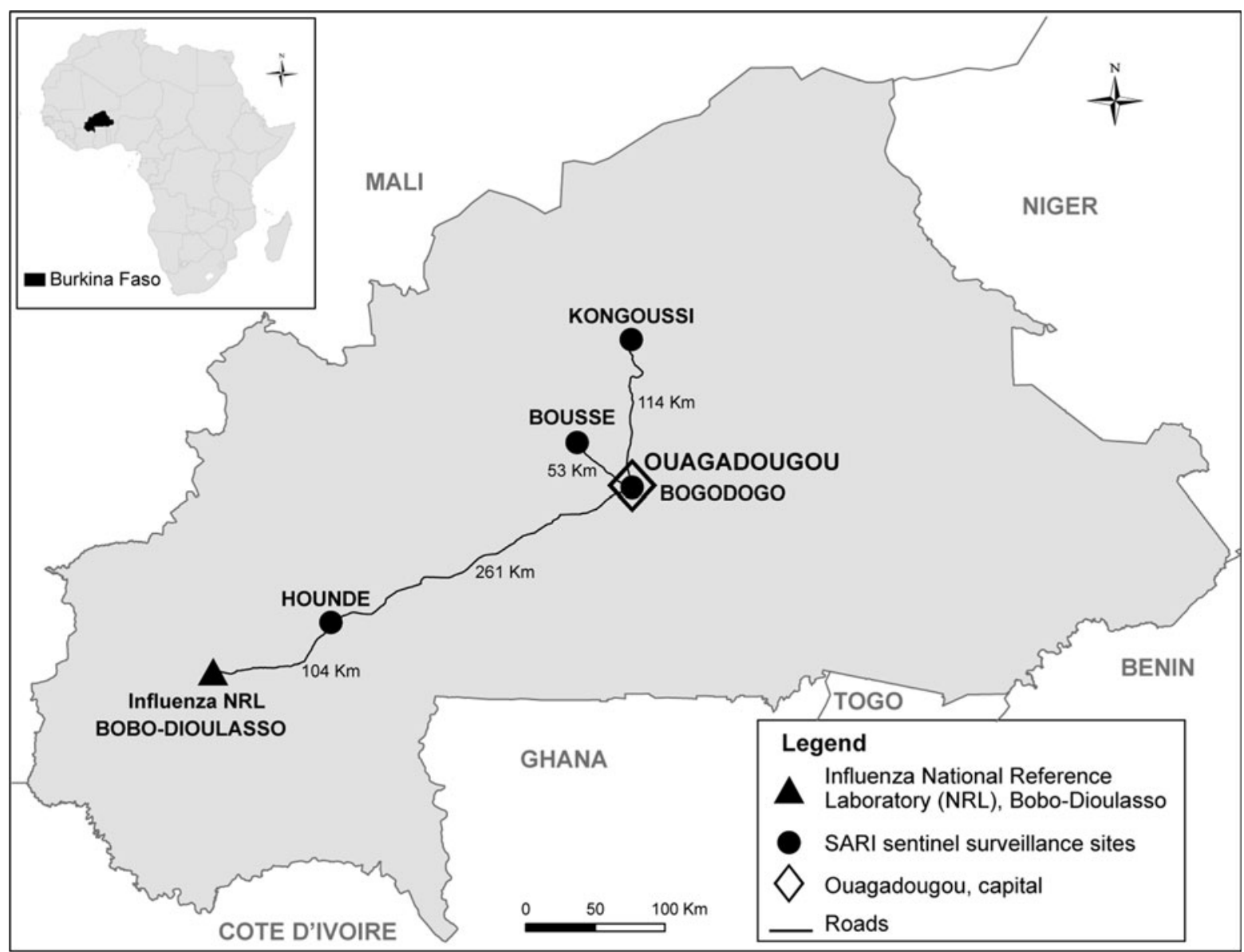

District hospitals included in the pilot specimen referral system included: Bogodogo, located in Ouagadougou, 365 kilometers from Bobo-Dioulasso; Houndé, in the Haut Bassins province, 104 kilometers from Bobo-Dioulasso; Boussé, in the Central Plateau, 419 kilometers from Bobo-Dioulasso; and Kongoussi, in the Central North province, 379 kilometers from BoboDioulasso.

Figure 1. Location of the severe acute respiratory infection sentinel surveillance sites districts: Bogodogo, Boussé, Kongoussi, and Houndé, Burkina Faso

also interviewed. Contextual information was solicited from CDC's Burkina Faso country office staff and country nongovernmental health partners, including Davycas International and John Snow, Inc. Discussions were conducted with logistics companies, including the national postal system (Sonapost), the National Federation of Road Transportation (FENAT), and Courtage International, a private courier company based in Burkina Faso.

\section{Baseline Assessment Findings}

Existing specimen transport systems were fragmented, with various disease surveillance systems using different transport modalities and funding sources. We found different routine specimen transport systems for 4 diseases or conditions under surveillance: measles, meningitis, human immunodeficiency virus (HIV) in infants, and yellow fever. The meningitis specimen transport system is more developed than the others, likely due to a successful, robust meningitis surveillance system that was instituted nationally in 2011. All 4 specimen transport systems were uniform in the transportation mode commonly used: biomedical technicians transported specimens via motorcycles from the health facility to the district laboratory and then via public transport (commercial bus companies) to Ouagadougou. From there, specimens traveled to an NRL either in Ouagadougo or Bobo-Dioulasso for testing.

Each specimen transport system used a different financing mechanism, making comparison of costs challenging. The estimated cost of transporting 1 specimen from the health facility to the reference laboratory using laboratory staff ranged from US $\$ 32$ to US $\$ 110$ and varied by the specimen type and distance traveled. This cost estimate included transportation fees and per diem for the laboratory staff responsible for the specimen in transit.

We also found that, rather than consolidating multiple specimens from 1 health facility and sending them together in the same package, several shipments were transported by different laboratory staff, thereby significantly increasing 
the overall cost of this method. This practice also took the health facility laboratory staff away from the laboratory for 1 to 2 days for each delivery, resulting in the loss of time spent working on regular laboratory duties. Incorrect packaging (not the required triple packaging), insufficient labeling (identification numbers not recorded on the tube), and delays in sending were additional issues identified in the assessment.

\section{Pilot Design Phase}

The ministry of health used an inclusive and participatory approach for the pilot specimen transport system design and implementation. Key stakeholders were consulted on the specimen transport system requirements for SARI surveillance, and this information supplemented the assessment findings to inform the specimen transport system design. To optimally serve the SARI surveillance system needs, the new specimen transport system had to include the following: (1) a schedule of pickups and dropoffs to meet the goal of specimen receipt at the influenza NRL within 48 hours of specimen collection; (2) identification of staff involved at each point in the system, and their roles and responsibilities; (3) implementation of relevant training, procedures, and communication networks; (4) supplies necessary for proper specimen collection, triplicate packaging, and transport; (5) a monitoring and evaluation plan; and (6) long-term financial and operational sustainability.

A request for proposals and terms of reference for transport and logistics services for the specimen transport system, based on models available through the Global Laboratory Initiative (GLI) specimen referral toolkit, were developed by the ministry of health, CDC, Davycas, ASLM, influenza NRL, and the specimen referral expert ${ }^{14}$ (see supplemental materials at https://www.liebertpub. com/doi/suppl/10.1089/hs.2019.0068). The request for proposals and terms of reference were approved at a national laboratory managers' meeting and published in the local newspaper in early 2017. The terms of reference required the service provider to transport specimens 3 times per week from the 4 sites to the influenza NRL, arriving within 24 to 48 hours of pickup, and to return the empty cooler boxes to the sites.

The ministry of health and partners received 4 proposals for review. The contract was awarded to Sonapost, the national postal system, for delivery using their Express Mail service. National coverage, expertise in logistics, experience in express package delivery, and reasonable cost of services made Sonapost the best candidate. In addition to using public transport, Sonapost has access to their own fleet of vehicles, including motorcycles, providing additional flexibility for the specimen transport system on the number of packages to be transported and distance. Although Sonapost lacked experience in transporting specimens, they were looking for opportunities to broaden their services, especially with a reduction in demand for their traditional mail services following the expansion of internet access in the country. Price was negotiated on a volume basis, regardless of specimen type, starting at approximately US\$3,200 per month for up to 100 packages, with unit costs decreasing as the shipment volumes increase to allow for cost efficiencies as additional specimen types are integrated. A contract was negotiated between Sonapost and Davycas on behalf of the partners, and implementation funding was provided via CDC through the GHSA program. ${ }^{2}$

In addition to Sonapost's contractual agreement, other partners had specific roles and responsibilities. The ministry of health was the overall technical lead, and ASLM provided technical guidance through the expert and in-country support for project execution, along with Davycas. Davycas was also responsible for contract management with Sonapost, setup, and supply costs. A detailed work plan with phased implementation and a monitoring and evaluation framework with specimen transport system performance indicators was developed. The specimen transport system start date was April 2017.

\section{Pilot Implementation Phase}

Standard operating procedures for sample packaging and collection were developed using WHO regulations for the biosafety of transporting infectious substances, and they were reviewed and approved by the ministry of health. ${ }^{6}$ To maintain the cold chain and ensure specimen quality upon arrival at the influenza NRL, specimens were triple packaged with 4 icepacks. Partners jointly trained staff at the 4 SARI sites along with 8 Sonapost staff members from the districts. The training covered the SOPs, case notification forms, and specimen tracking forms. Refresher training sessions were conducted with Sonapost staff periodically because of staff turnover. Sonapost national-level staff received training from ASLM and Davycas on biosafety and then trained their agents and transporters at corresponding district, provincial, and regional offices.

Each site received a core set of packaging and shipping supplies: triple packaging materials (plastic bags, bubble wrap, tape, cotton, plastic cylinder) to hold the SARI specimens, cooler boxes, ice packs, a plastic sleeve for specimen and case report forms when placed in the cooler box, and biohazard stickers. These were monitored by sites and replaced as needed. The ministry of health surveillance team and partners conducted regular monitoring at each site along with periodic refresher training on triplicate packaging. If the influenza NRL observed a problem related to packaging or specimen quality, the surveillence site was immediately notified to take corrective action. Data to track specimen movement (collection date and time, delivery to the district laboratory, Sonapost pickup and receipt at influenza NRL, and condition) were recorded on the case 
report form that accompanied the specimen. The influenza NRL assigned a unique identification number to each specimen received and recorded the surveillance identifier from the case notification form. These data were used to monitor the time to receipt and specimen quality when received at the influenza NRL.

A direct line of communication was established among the laboratory unit head at each surveillance site, respective district Sonapost agent, and influenza NRL to ensure monitoring and evaluation data were being collected and used for continuous quality improvement. Key performance indicators included the: (1) increase in use of laboratorybased surveillance (measured by number of packages and specimens delivered), (2) timeliness of specimen delivery (measured by the percentage of specimens delivered within 24 hours of pickup by Sonapost and within 48 hours of sample collection), and (3) quality of courier services (measured by the number of packages missing or damaged during transit).

As part of SARI surveillance monitoring, the specimen transport data quality were assessed in February 2019 at the 4 sentinel sites and at the influenza NRL. A 3-day audit was conducted at each site by a team composed of Davycas International, the ministry of health, CDC, and Sonapost staff. The team worked with site staff to review the site database for data on specimen collection and movement, to cross-check data with the site laboratory logbook, and to triangulate with data on date, time, and package number in the Sonapost database. Data were confirmed, and all dis- crepancies were corrected and recorded in a central ministry of health Epinfo database, and EpiInfo and Excel software were used for all analyses.

\section{Results}

From May 2017 to December 2018, 557 packages containing a total of 1,158 SARI specimens were transported from the 4 district surveillance sites to the influenza NRL by Sonapost. No packages were reported missing or lost by the surveillance sites, influenza NRL, or Sonapost. Of the 557 packages, 95\% (529/557) were delivered to the influenza NRL within 24 hours of pickup at the surveillance site, and $77 \%(892 / 1,158)$ of specimens arrived at the influenza NRL within 48 hours of collection from the patient. Both indicators met the targets in the WHO guidelines, which recommend that SARI specimens be processed within 48 to 72 hours of collection. ${ }^{13}$ We found that $41 \%(475 / 1,158)$ of specimens were transported from November 2017 to March 2018. This timeframe corresponds with an increase in cases detected by the SARI surveillance system, indicating an increase in specimen transport system use during the respiratory disease season (Figure 2). The range of specimens per package was 1 to 10 , with an average of 2 per package.

The average time from Sonapost pickup to receipt at the influenza NRL varied by surveillance site. The site with the lowest proportion of specimens arriving at the influenza NRL within 24 hours of pickup was Kongoussi. The

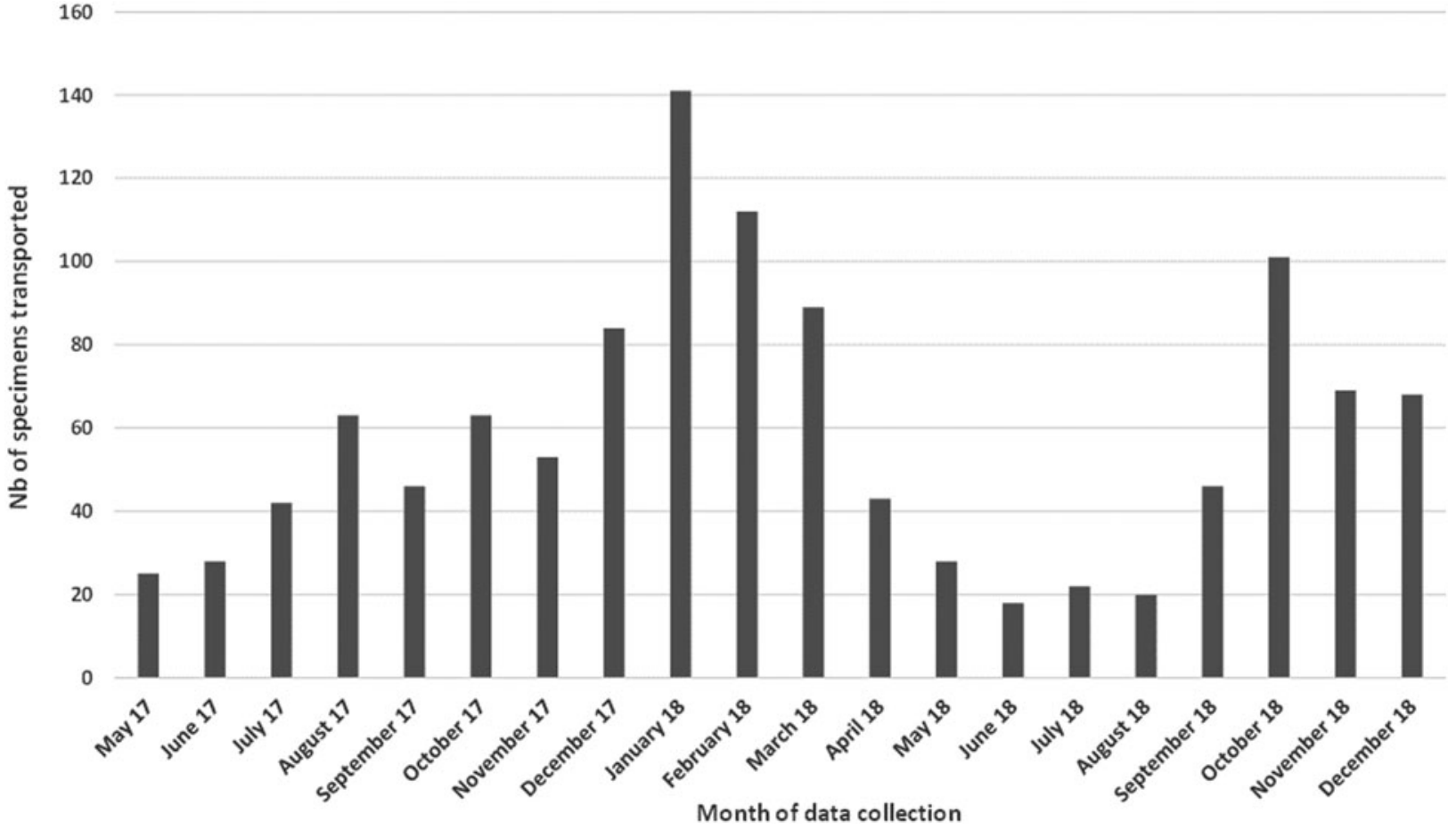

Figure 2. Number of severe acute respiratory infection specimens transported via the pilot specimen transport system to the influenza National Reference Laboratory, May 2017-December 2018, Burkina Faso 

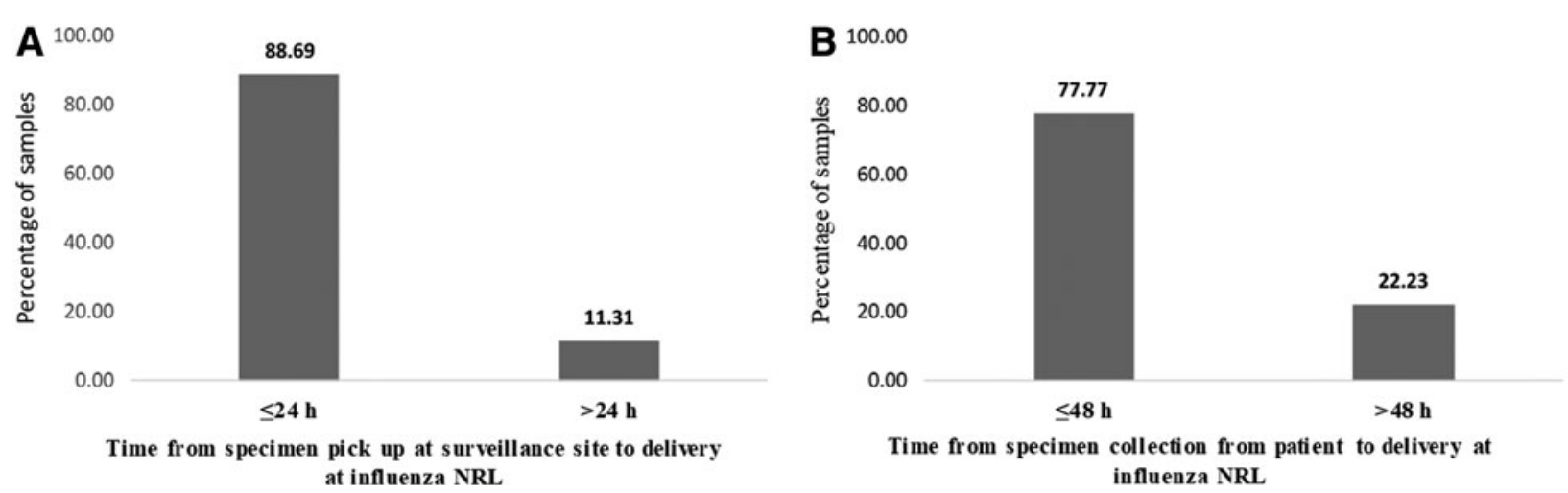

Figure 3. Distribution of severe acute respiratory illness surveillance samples received at influenza National Reference Laboratory from (A) specimens pickup at surveillance site, and (B) from specimen collection from patient between May 2017 and December 2018, Burkina Faso

surveillance site closest to the influenza NRL, Hounde, had $100 \%$ of specimens arrive within 24 hours of package collection; $89 \%$ of all samples were received in the influenza NRL the day following pickup, while $11 \%$ reached the influenza NRL the same day (Figure 3). Specimen quality review on receipt at the influenza NRL found that all specimens were received in good condition. In the event a package could not be transported within 24 to 48 hours to the influenza NRL, the Sonapost agents maintained them in cold storage in Ouagadougou until they could be safely transported.

\section{Discussion}

We found that the newly implemented specimen transport system in Burkina Faso was able to reliably deliver SARI surveillance specimens in good condition within 24 to 48 hours to the influenza NRL, even from the most distant surveillance site, with no packages lost. We saw an increase in the volume of specimens transported during the respiratory season and reductions in transport time with the specimen transport system establishment.

Postal services have been used to transport tuberculosis (TB) and HIV specimens in Uganda and Ethiopia, where the system was based on a public-private partnership. Our findings of increased proportion of specimens transported and reduced transportation time using the postal system were similar to previous studies. ${ }^{15,16}$ Colleagues in Guinea also worked to establish a specimen referral and transport system following the West Africa Ebola virus disease outbreak, and they have documented the importance of collaborating closely with the ministry of health and conducting a baseline assessment to inform system design. ${ }^{17}$

The specimen transport system pilot had some significant limitations. It was challenging to estimate the cost benefits achieved with the new specimen transport system. A quantitative comparison of the specimen transport system using Sonapost with the previous model, where laboratory staff transported specimens themselves, is difficult, as we do not have specific data on the time staff spent carrying specimens and away from their regular laboratory duties. However, the previous system was likely more expensive, as it used extensive staff time and required staff per diems and took staff away from their usual duties, delaying laboratory workflow. While the cost per package of the specimen transport system with Sonapost was relatively high, as it was piloted for 1 surveillance system, the inclusion of additional surveillance systems will create economies of scale that could result in lower costs per package.

Because the specimen transport system was piloted using a public health surveillance system, required activities did not include the return of test results to the clinical sites. If additional surveillance systems participate in the specimen transport system, and testing for clinical use is included (as for HIV and TB), it will be important to redesign the monitoring and evaluation framework and indicators to include the return of test results to patients.

Although the specimen transport system was designed to support SARI surveillance, it has already proven to be adaptable to other disease surveillance systems and specimen types in Burkina Faso. In August 2017, the country experienced a dengue outbreak that required the rapid referral of blood specimens from suspect cases in Ouagadougou to the NRL for arboviruses at Centre Muraz in Bobo-Dioulasso. The ministry of health leveraged the specimen transport system for this purpose, and the specimens arrived in good condition and within the time frame required for testing and reporting. This specimen transport system did not incur any additional fees, as the number of total shipments remained within the current price band of up to 100 packages monthly. ${ }^{18}$

Discussions are currently under way to incorporate this specimen transport system model into additional disease surveillance systems, to expand the specimen types included, and to expand the system nationally. If individual disease surveillance programs pool resources currently being used to support multiple vertical specimen transport systems, we believe a national specimen transport system may be feasible. 
In the future, the ministry of health and partners will need to devise strategies to bring together donors and disease surveillance systems to participate and contribute to a fully integrated specimen transport system. Demonstration of cost efficiency will contribute important evidence to this effort. Sonapost remains committed to the activities and is actively engaged with the ministry of health in training on safe transport and direct communication with stakeholders. Key lessons for other countries interested in developing similar systems are the importance of stakeholder engagement throughout the process; implementation of standardized procedures, training, and communication systems at all specimen transport system phases; and monitoring and evaluation framework use with measurable indicators to monitor routine progress. Burkina Faso's experience demonstrated a feasible approach for bringing together multiple partners to develop a standardized specimen transport system that has the potential to integrate other diseases and enhance both laboratory capacity and public health infrastructure, thereby enhancing global health security implementation.

\section{REFERENCES}

1. World Health Organization. The Review Committee on the Role of the International Health Regulations (2005) in the Ebola Outbreak and Response. May 13, 2016. https://www. who.int/ihr/review-committee-2016/en/. Accessed October 21, 2019.

2. Tappero JW, Cassell CH, Bunnell RE, et al. US Centers for Disease Control and Prevention and its partners' contributions to global health security. Emerg Infect Dis 2017;23(Suppl):S5-S14.

3. Fitzmaurice AG, Mahar M, Moriarty LF, et al. Contributions of the US Centers for Disease Control and Prevention in implementing the Global Health Security Agenda in 17 partner countries. Emerg Infect Dis 2017;23(Suppl):S15-S24.

4. Nkengasong JN, Mesele T, Orloff S, et al. Critical role of developing national strategic plans as a guide to strengthen laboratory health systems in resource-poor settings. $A m J$ Clin Pathol 2009;131(6):852-857.

5. Nkengasong JN. Strengthening laboratory services and systems in resource-poor countries. Am J Clin Pathol 2009;131(6):774.

6. World Health Organization. Guidance on regulations for the transport of infectious substances 2015-2016. 2015. https:// www.who.int/ihr/publications/who_hse_ihr_2015.2/en/. Accessed October 21, 2019.

7. United Nations. European agreement concerning the international carriage of dangerous goods by road. United $\mathrm{Na}-$ tions Economic Commission for Europe (UNECE). 2017. https://www.unece.org/trans/danger/publi/adr/adr_e.html. Accessed October 21, 2019.
8. Bonkoungou IJO, Aliabadi N, Leshem E, et al. Impact and effectiveness of pentavalent rotavirus vaccine in children $<5$ years of age in Burkina Faso. Vaccine 2018;36(47):71707178.

9. Kyelem CG, Bougouma A, Thiombiano RS, et al. Cholera epidemic in Burkina Faso in 2005: epidemiologic and diagnostic aspects. Pan Afr Med J 2011;8:1.

10. Sagna T, Ilboudo AK, Wandaogo C, et al. Preliminary results of official influenza and acute respiratory infection surveillance in two towns of Burkina Faso, 2013-2015. BMC Infect Dis 2018;18(1):330.

11. Whaley MJ, Jenkins LT, Hu F, et al. Triplex real-time PCR without DNA extraction for the monitoring of meningococcal disease. Diagnostics 2018;8(3):58.

12. Yaesoubi R, Trotter C, Colijn C, et al. The cost-effectiveness of alternative vaccination strategies for polyvalent meningococcal vaccines in Burkina Faso: a transmission dynamic modeling study. PLoS Med 2018;15(1):e1002495.

13. World Health Organization. Global epidemiological surveillance standards for influenza. WHO website. January 2014. https://www.who.int/influenza/resources/documents/ influenza_surveillance_manual/en/. Accessed October 22, 2019.

14. Global Laboratory Initative. GLI Guide to TB specimen referral systems and integrated networks. Stop TB Partnership. 2018. http://www.stoptb.org/wg/gli/assets/documents/ gli_guide_specimens_web_ready.pdf. Accessed October 21, 2019.

15. Sanou AS, Dirlikov E, Sondo KA, et al. Building laboratorybased arbovirus sentinel surveillance capacity during an ongoing dengue outbreak, Burkina Faso, 2017. Health Secur 2018;16(Suppl):S103-S110.

16. Joloba M, Mwangi C, Alexander H, et al. Strengthening the tuberculosis specimen referral network in Uganda: the role of public-private partnerships. J Infect Dis 2016;213(Suppl 2): S41-S46.

17. Kebede Y, Fonjungo PN, Tibesso G, et al. Improved specimen-referral system and increased access to quality laboratory services in Ethiopia: the role of the public-private partnership. J Infect Dis 2016;213(Suppl 2):S59-S64.

18. Standley CJ, Muhayangabo R, Bah MS, et al. Creating a national specimen referral system in Guinea: lessons from initial development and implementation. Front Public Health 2019;7:83.

Address correspondence to: Emilie Dama, PhD Centers for Disease Control and Prevention Prevention Research Centers American Embassy secteur 15, Ouaga 2000 Rue 15.873 Ouagadougou 35 Burkina Faso

Email: DamaE@state.gov 